\title{
Dringliche Gesetzgebung und direkte Demokratie
}

\section{Ein Blick auf Grundlagen, Praxis und Probleme des nachträglichen Referendums im Bund anlässlich der Volksabstimmung vom 13. Juni 2021 über das Covid-19-Gesetz}

\begin{abstract}
Die Notwendigkeit, gesetzgeberisch rasch zu handeln, und die Forderung, die direktdemokratischen Rechte möglichst weitgehend zu wahren, führen im Bereich des Dringlichkeitsrechts zu einem Spannungsverhältnis. Das 1949 aufgrund einer Volksinitiative eingeführte nachträgliche Referendum im Bund dient dessen Auflösung. Die Autoren skizzieren Grundlagen und Praxis dieser weniger bekannten Referendumsart und befassen sich mit den besonderen Problemen, welche die Volksabstimmung vom 13. Juni 2021 über das Covid19-Gesetz stellt.
\end{abstract}

Beitragsart: Wissenschaftliche Beiträge

Rechtsgebiete: Politische Rechte; Staatsorganisation und Behörden 


\section{Inhaltsübersicht}

1. Einleitende Bemerkungen

2. Dringlichkeitsrecht und Referendum: Grundlagen

2.1. Die Einführung des nachträglichen Referendums 1949

2.2. Die Regelung des nachträglichen Referendums nach geltendem Recht

3. Dringlichkeitsrecht und Referendum: Praxis

3.1. Die Konjunkturen des Dringlichkeitsrechts

3.2. Die bisherigen Volksabstimmungen im Überblick

4. Probleme des Referendums gegen das Covid-19-Gesetz

4.1. Die Ausgangslage

4.2. Der Gegenstand der Volksabstimmung

4.3. Die Wirkungen einer allfälligen Ablehnung des Gesetzes

4.3.1. Schicksal des Covid-19-Gesetzes

4.3.2. Schicksal der wirtschaftlichen Unterstützungsmassnahmen

5. Zusammenfassende Schlussfolgerungen

\section{Einleitende Bemerkungen}

[1] Am 12. Januar 2021 reichte ein Referendumskomitee rund um die Gruppierung «Freunde der Verfassung» knapp 100'000 Unterschriften gegen das Covid-19-Gesetz ${ }^{1}$ ein, das die Bundesversammlung am 25. September 2020 erlassen hatte, um eine gesetzliche Grundlage für Massnahmen der Pandemiebekämpfung und der Abfederung der wirtschaftlichen Auswirkungen zu schaffen. Die Bundeskanzlei stellte am 2. März 2021 fest, das Referendum sei zustande gekommen. ${ }^{2}$

[2] Die Bundesversammlung hatte das Gesetz dringlich im Sinn von Art. 165 Abs. 1 BV erklärt. Es trat damit nicht wie im ordentlichen Gesetzgebungsverfahren erst nach Ablauf der 100-tägigen Referendumsfrist gemäss Art. 141 Abs. 1 BV, sondern unter Vorbehalt der sogar rückwirkend auf den 17. September 2020 in Kraft gesetzten Bestimmung über Massnahmen zur Entschädigung des Erwerbsausfalls (vgl. Art. 21 Abs. 3 Covid-19-Gesetz) bereits am folgenden Tag in Kraft (vgl. Art. 21 Abs. 1 und 2 Covid-19-Gesetz).

[3] Das Referendum kann in einem solchen Fall also nur eine resolutive, abrogative, d.h. aufhebende, und keine suspensive, aufschiebende Wirkung entfalten. ${ }^{4}$ Diese besondere, auf nationaler Ebene vor allem in Italien bekannte Referendumsart ${ }^{5}$ kann in der Praxis schwierige demokra-

1 Bundesgesetz vom 25. September 2020 über die gesetzlichen Grundlagen für Verordnungen des Bundesrates zur Bewältigung der Covid-19-Epidemie (SR 818.102).

2 Vgl. Verfügung der Bundeskanzlei vom 2. März 2021 über das Zustandekommen des Referendums gegen das Bundesgesetz vom 25. September 2020 über die gesetzlichen Grundlagen für Verordnungen des Bundesrates zur Bewältigung der Covid-19-Epidemie (Covid-19-Gesetz) (BBl 2021 460); von den eingereichten knapp 100'000 Unterschriften wurden jedoch 70'000 ohne Stimmrechtsbescheinigung eingereicht. Das Referendumskomitee profitierte demnach ironischerweise von demjenigen Gesetz, welches es bekämpfen möchte. Art. 2 Covid-19-Gesetz ermöglicht nämlich vorübergehend die Einreichung von Unterschriftenlisten ohne vorgängige Bescheinigung (vgl. hinten 4.3.1). Ob das Referendum ohne diese wesentliche Erleichterung überhaupt zustande gekommen wäre, ist fraglich.

3 Bundesverfassung der Schweizerischen Eidgenossenschaft vom 18. April 1999 (BV; SR 101).

4 Vgl. statt vieler Pierre Tschannen, in: Bernhard Ehrenzeller/Benjamin Schindler/Rainer J. Schweizer/Klaus A. Vallender (Hrsg.), Die schweizerische Bundesverfassung, St.Galler Kommentar, 3. Aufl., Zürich/St.Gallen 2014 (zit. SGK-Autor), Art. 165 N. 14; zum Begriff des abrogativen Referendums auch René Rhinow/Markus Schefer/Peter Uebersax, Schweizerisches Verfassungsrecht, 3. Aufl., Basel 2016, N. 2203; ferner Corsin Bisaz, Direktdemokratische Instrumente als «Anträge aus dem Volk an das Volk», Habil. Zürich 2020, N. 357.

5 In Italien können 50’000 Wahlberechtigte oder fünf Regionalräte die Aufhebung eines Gesetzes bzw. einer Gesetzesänderung verlangen. Die Referendumsvorlage gilt als angenommen, wenn mindestens die Hälfte der Stim- 
tierechtliche und -politische Fragen aufwerfen. Das zeigt sich beispielhaft im aktuellen Fall, der nachfolgend im Kontext der bisherigen Referendumspraxis im Bereich des Dringlichkeitsrechts beleuchtet wird.

\section{Dringlichkeitsrecht und Referendum: Grundlagen}

\subsection{Die Einführung des nachträglichen Referendums 1949}

[4] Die Behauptung, direkte Demokratie tauge nur bei schönem Wetter, nicht aber in stürmischen Zeiten, gehört zu den klassischen Argumenten ihrer Kritiker. ${ }^{6}$ Tatsächlich stehen in Krisensituationen die Notwendigkeit raschen Handelns und die Forderung direktdemokratischer Legitimation in einem Spannungsverhältnis. Art. $89 \mathrm{aBV}^{7}$ enthielt in der ursprünglichen Fassung denn auch eine Bestimmung, wonach Bundesgesetze und «allgemein verbindliche Bundesbeschlüsse, die nicht dringlicher Natur sind», auf Verlangen von 30'000 Stimmberechtigten oder von acht Kantonen der Volksabstimmung zu unterstellen waren (Art. 89 Abs. 2 aBV; Hervorhebung hinzugefügt). Der Begriff des «allgemein verbindlichen Bundesbeschlusses» blieb allerdings ein stets umstrittenes «Sorgenkind vieler Generationen von Staatsrechtlern» ${ }^{8}$. Die Beantwortung der Frage, was alles unter diesen Begriff fallen sollte, war deshalb von entscheidender Bedeutung, weil von ihr sowohl das «Schicksal» des Verwaltungsreferendums auf Bundesebene (d.h. eines Referendums über Verwaltungsbeschlüsse, insbesondere auch Ausgabenbeschlüsse der Bundesversammlung in der Form des allgemeinverbindlichen Bundesbeschlusses) ${ }^{9}$ als auch der mögliche Umfang des Dringlichkeitsrechts abhing. Die erwähnte Verfassungsbestimmung erlaubte nämlich $e$ contrario die Dringlicherklärung von allgemeinverbindlichen Bundesbeschlüssen, mit der Folge, dass diese dem fakultativen Referendum entzogen waren. ${ }^{10}$ Tatsächlich ergingen zwischen 1919 und 1938148 dringliche Bundesbeschlüsse, während im gleichen Zeitraum nur 145 nicht dringliche Bundesbeschlüsse sowie Bundesgesetze verabschiedet wurden. Während zwischen 1919 und 1928 noch mehr nicht dringliche als dringliche Erlasse zustande kamen, verkehrte sich zwischen 1929 und 1938 die Ausnahme in die Regel; zwischen 1934 und 1938 waren es gar doppelt so viele dringliche (56) als nicht dringliche (28) Bundesbeschlüsse, ${ }^{11}$ was unter dem Gesichtspunkt der direkten Demokratie zu einem «Teilzusammenbruch des politischen Systems» ${ }^{12}$ führte und

menden dafür stimmt, wobei die Stimmbeteiligung mindestens 50 Prozent betragen muss (Art. 75 Abs. 1 und 4 Costituzione della Repubblica Italiana vom 27. Dezember 1947). Sodann kann in Dänemark eine Parlamentsminderheit verlangen, dass eine nachträgliche, abrogativ wirkende Volksabstimmung über dringliche Gesetze durchgeführt wird (§ 42 Abs. 7 Danmarks Riges Grundlov vom 5. Juni 1953). Vgl. zum Ganzen Walter HaLleR/ Alfred Kölz/Thomas Gächter, Allgemeines Staatsrecht, 6. Aufl., Zürich/Basel/Genf 2020, N. 272, 302 f.

6 Vgl. auch Antoine ChOlLet, Défendre la démocratie directe, Sur quelques arguments antidémocratiques des élites suisses, Lausanne 2011, S. $91 \mathrm{f}$.

7 Bundesverfassung der Schweizerischen Eidgenossenschaft vom 29. Mai 1874 (aBV; AS 1 [1874] 1).

8 Alfred Kölz, Neuere schweizerische Verfassungsgeschichte, Bd. 2: Ihre Grundlinien in Bund und Kantonen seit 1848 , Bern 2004, S. 617.

9 Vgl. Kölz (Fn. 8), S. 617.

10 Vgl. Stefan G. Schmid, Entstehung und Entwicklung der Demokratie in der Schweiz, in: Oliver Diggelmann/Maya Hertig Randall/Benjamin Schindler (Hrsg.), Verfassungsrecht der Schweiz, Zürich 2020, Bd. 1, II.1, N. 24.

11 Zahlen bei Conradin Regi, Die Grenzen des fakultativen Referendums in der Bundesgesetzgebung, Diss. Bern, Chur 1945, S. 56.

12 Kölz (Fn. 8), S. 767. 
die Referendumsdemokratie im Bund «weitgehend entscheidungsunfähig» ${ }^{13}$ machte. Bundesrat und Bundesversammlung verfolgten in den 1930er-Jahren, ähnlich wie Präsident Franklin Delano Roosevelt mit seiner «New Deal-Gesetzgebung» in den USA, ${ }^{14}$ mit dem Dringlichkeitsrecht eine interventionistische, wohlfahrtsstaatliche Politik - zu den drastischen Massnahmen zählte etwa das Verbot der Eröffnung und Erweiterung von Warenhäusern und Einheitspreisgeschäften $-{ }^{15}$ zur Lösung der von der Weltwirtschaftskrise verursachten wirtschaftlichen und sozialen Probleme. ${ }^{16}$ Die dringlichen Bundesbeschlüsse kamen allerdings häufig ohne zeitliche Dringlichkeit zustande, wurden oft nicht oder nicht richtig begründet und alle zwei Jahre um weitere zwei Jahre verlängert. ${ }^{17}$ Ein grosser Teil der wirtschaftsrechtlichen Bundesbeschlüsse widersprach nicht nur der Handels- und Gewerbefreiheit (Grundsatz der Wettbewerbswirtschaft), sondern auch der bundesstaatlichen Kompetenzverteilung und blieb, obwohl nicht bloss vorübergehender Natur, dem Referendum entzogen; sie waren damit «auf dreifache Weise nicht verfassungskonform». ${ }^{18}$ Dabei spielte das Motiv, mit der Wahl des Dringlichkeitsrechts ein Referendum zu vermeiden, in der Bundesversammlung eine wichtige Rolle. ${ }^{19}$ Die auch in der Wissenschaft kritisierte Praxis ${ }^{20}$ - der Zürcher Staatsrechtler ZACCARIA Giacometti sprach von einer «illegale[n] Praxis», ${ }^{21}$ von «autoritäre[m] Bundesstaatsrecht», «Parlamentsabsolutismus» und «Parlamentsdiktatur» ${ }^{22}$ - provozierte verschiedene Volksinitiativen, die insbesondere darauf abzielten, das Referendumsrecht und die Verfassungsgerichtsbarkeit zu stärken. ${ }^{23}$ So lancierten die Sozialdemokratische Partei und verschiedene Gewerkschaften die Volksinitiative betreffend «Einschränkung der Anwendung der Dringlichkeitsklausel», der die Bundesversammlung schliesslich einen direkten Gegenentwurf gegenüberstellte. ${ }^{24}$ Dieser fand 1939 die Zustimmung einer Mehrheit von Volk und Ständen und ergänzte Art. 89 aBV mit einem Absatz, wonach ausdrücklich nur Bundesbeschlüsse, deren Inkrafttreten keinen Aufschub ertrug, dringlich erklärt werden konn-

13 Leonhard Neidhart, Plebiszit und pluralitäre Demokratie, Eine Analyse der Funktion des schweizerischen Gesetzesreferendums, Diss. Berlin, Bern 1970, S. 231.

14 Vgl. Kölz (Fn. 8), S. 764.

15 Vgl. Andreas Kley, Verfassungsgeschichte der Neuzeit, Grossbritannien, die USA, Frankreich und die Schweiz, 4. Aufl., Bern 2020, S. 323; Kölz (Fn. 8), S. 764 ff.; eingehend dazu David Reich, Direkte Demokratie in der Krise, Die Funktion des Notrechts in der Schweiz während Weltwirtschaftskrise und Zweitem Weltkrieg dargestellt am Beispiel des Warenhausbeschlusses 1933-1945, Diss. Basel 2007.

19 Vgl. Hellmut HungerbüHler, Der dringliche Bundesbeschluss, Diss. Zürich 1951, S. 92 ff., mit einer aufschlussreichen Zusammenstellung von Voten aus dem Stenographischen Bulletin.

20 Vgl. Andreas KLey, Von Stampa nach Zürich, Der Staatsrechtler Zaccaria Giacometti, sein Leben und Werk und seine Bergeller Künstlerfamilie, Zürich/Basel/Genf 2014, S. 248 ff.; DERs., Geschichte des öffentlichen Rechts der Schweiz, 2. Aufl., Zürich/St. Gallen 2015, S.175 f.; KöLz (Fn. 8), S. 768 ff.

21 Zaccaria Giacometti, Die Verfassungsmässigkeit der Bundesgesetzgebung und ihre Garantien, SJZ 30 (1933/34), S. 289 ff., S. 290. Vgl. dazu Kley, Giacometti (Fn. 20), S. 248 ff.

22 Zaccaria Giacometti, Verfassungsrecht und Verfassungspraxis in der schweizerischen Eidgenossenschaft (Das autoritäre Bundesstaatsrecht), in: Rechts- und staatswissenschaftliche Fakultät der Universität Zürich (Hrsg.), Festgabe Fritz Fleiner, Zürich 1937, S. 45 ff., S. 46 f. (alle drei Zitate). Vgl. dazu Kölz (Fn. 8), S. 768.

23 Vgl. KLEY, Geschichte des öffentlichen Rechts (Fn. 20), S. 168 ff., 173 Fn. 1094; KöLz (Fn. 8), S. 771 ff.; REICH (Fn. 15), S. 202 ff., 260 ff.

24 Die Volksinitiative wurde in der Folge zurückgezogen (BB1 1938 II 1205). 
ten, und zwar durch die Mehrheit aller Mitglieder in jedem der beiden Räte. Ferner waren die dringlichen allgemeinverbindlichen Bundesbeschlüsse zu befristen. ${ }^{25}$

[5] Diese Neuregelung des Dringlichkeitsrechts vermochte allerdings nie richtig Wirkung zu entfalten, vor allem deshalb, weil schon rund sieben Monate später mit Ausbruch des Zweiten Weltkriegs der Vollmachtenbeschluss der Bundesversammlung erging, ${ }^{26}$ der die Verfassungsordnung teilweise suspendierte. ${ }^{27}$ Nach Kriegsende lancierten Waadtländer Freisinnige und Liberale «aus einer Kombination von rechtsfreisinnigem Anti-Etatismus und welschem Föderalismus» ${ }^{28}$ die von Bundesversammlung und Bundesrat - zuweilen in einem «stark landesväterlich polizeistaatlichen Ton» ${ }^{29}$ - bekämpfte Volksinitiative «für die Rückkehr zur direkten Demokratie», die - als einzige Volksinitiative zwischen 1929 und 1982 - 1949 sehr knapp (von 50.7 Prozent der Stimmenden und $113 / 2$ Ständen) angenommen wurde. ${ }^{30}$ Der neu eingefügte Art. $89^{\text {bis }}$ aBV durchbrach nun erstmals die repräsentative Demokratie in der dringlichen Rechtsetzung durch eine Einrichtung direkter Demokratie, indem sie ein nachträgliches Referendum in der Bundesverfassung verankerte: ein fakultatives bei verfassungskonformen und ein obligatorisches bei nicht verfassungskonformen dringlichen Bundesbeschlüssen; in beiden Fällen entgingen diese in der Praxis allerdings dem Referendum, wenn die Bundesversammlung sie für weniger als ein Jahr in Kraft setzte. ${ }^{31}$ Für den Fall einer Ablehnung wurde vorgesehen, dass ein solcher Beschluss ausser Kraft treten müsse und nicht erneuert werden könne. Diese Regelung wurde - unter Ersetzung des allgemeinverbindlichen Bundesbeschlusses durch das Bundesgesetz - in den heute geltenden Art. 165 BV übernommen. Neben dem Bund kennt auch knapp die Hälfte der Kantone ein nachträgliches Referendum im Bereich der dringlichen Gesetzgebung. ${ }^{32}$

25 Vgl. Art. 89 Abs. 3 aBV, angenommen in der Volksabstimmung vom 22. Januar 1939 (BB1 1939 I 161; AS 55 [1939] 242); Abs. 3 betreffend das Staatsvertragsreferendum wurde neu zu Abs. 4. Vgl. zum Ganzen KLey, Verfassungsgeschichte (Fn. 15), S. 354; KöLz (Fn. 8), S. 771.

26 Vgl. Bundesbeschluss vom 30. August 1939 über Massnahmen zum Schutze des Landes und zur Aufrechterhaltung der Neutralität (AS 1939 769); Näheres bei KLEY, Geschichte des öffentlichen Rechts (Fn. 20), S. 192 ff.; KLEY, Verfassungsgeschichte (Fn. 15), S. 333 ff.; Kölz (Fn. 8), S. 773 ff. Vgl. KLEY, Verfassungsgeschichte (Fn. 15), S. 354.

28 Georg Kreis, Parlamentarismus und Antiparlamentarismus in den Jahren 1933-1945, in: Schweizerische Parlamentsdienste (Hrsg.), Das Parlament - «Oberste Gewalt des Bundes»?, Bern/Stuttgart 1991, S. 301 ff., S. 319 Fn. 60

Fritz Fleiner/Zaccaria Giacometti, Schweizerisches Bundesstaatsrecht, Zürich 1949, S. 789 Fn. 7, betreffend den Bericht vom 27. Februar 1948 über das Volksbegehren vom 23. Juli 1946 für die Rückkehr zur direkten Demokratie (BB1 1948 I 1054).

30 Vgl. Art. $89^{\text {bis }}$ aBV, angenommen in der Volksabstimmung vom 11. September 1949 (BB1 1949 II 581 ; AS 1949 1511); dazu Etienne Grisel, Art. 89 ${ }^{\text {bis }}$, in: Jean-François Aubert/Kurt Eichenberger/Jörg Paul Müller/René A. Rhinow/Dietrich Schindler (Hrsg.), Kommentar zur Bundesverfassung der Schweizerischen Eidgenossenschaft vom 29. Mai 1874, Loseblatt, Basel/Zürich/Bern 1987; zum Ganzen KLEY, Verfassungsgeschichte (Fn. 15), S. 354 f.; KöLz (Fn. 8), S. 780. Die Vermutung von KöLz (Fn. 8), S. 780, wonach Zaccaria Giacometti den Initiativtext verfasst haben soll, scheint aber nicht zuzutreffen (vgl. KLeY, Giacometti [Fn. 20], S. 278 Fn. 985; Rafael KüFfER, Eine liberale Kritik am Notrecht, Zaccaria Giacometti als Protagonist der Schweizer Notrechtsdebatte, Diss. Bern, Tübingen 2014, S. 173 f.; ferner Reich [Fn. 15], S. 260 Fn. 1224).

31 Vgl. Grisel, Kommentar aBV (Fn. 30), Art. $89^{\text {bis }}$ aBV, N. 21, 31; ferner etwa Jean-François Aubert, Bundesstaatsrecht der Schweiz, Neubearbeiteter Nachtrag bis 1994, Band II, Basel/Frankfurt a.M. 1995, N. 1126*; ULRICH HäFelin/Walter Haller, Schweizerisches Bundesstaatsrecht, 4. Aufl., Zürich 1998, N. 990. In der Lehre wurde diese Auslegung von Art. $89^{\text {bis }}$ aBV allerdings vereinzelt auch in Frage gestellt (vgl. den Hinweis bei Thomas GÄснтеR, Demokratie und Dringlichkeit, Gedanken zu Geschichte und Anwendung des Dringlichkeitsrechts der schweizerischen Bundesverfassung, in: Isabelle Häner [Hrsg.], Nachdenken über den demokratischen Staat und seine Geschichte, Beiträge für Alfred Kölz, Zürich/Basel/Genf 2003, S. 75 ff., S. 82).

32 Vgl. Andreas Auer, Staatsrecht der schweizerischen Kantone, Bern 2016, N. 677, 1016, 1024, je mit Nachweis der einschlägigen Verfassungsbestimmungen. Die Zahl der Kantone mit nachträglichem Referendum liegt aber höher als a.a.O., N. 1016 angegeben (vgl. auch Art. 18 Abs. 2 der Verfassung des Kantons Graubünden vom 18. Mai 


\subsection{Die Regelung des nachträglichen Referendums nach geltendem Recht}

[6] So essenziell die Einrichtung der dringlichen Bundesgesetzgebung in Krisensituationen sein mag, so problematisch ist sie aus demokratischer Sicht, da hierdurch das ordentliche Gesetzgebungsverfahren mitsamt seinen (direkt-)demokratischen Mitwirkungsrechten ${ }^{33}$ erheblich modifiziert, ja teilweise suspendiert wird.

[7] Um auch in Krisensituationen dem Spannungsverhältnis zwischen einerseits effizienter und andererseits demokratisch hinreichend legitimierter Gesetzgebung gerecht zu werden, müssen für die Verabschiedung dringlicher Bundesgesetze zwingend kumulativ folgende Voraussetzungen erfüllt sein (vgl. Art. 165 BV):

1. Qualifiziertes Beschluss-Quorum: Ein Bundesgesetz muss von der Mehrheit der Mitglieder der beiden Räte dringlich erklärt werden (vgl. auch Art. 159 Abs. 3 Bst. a BV), d.h. von 101 Mitgliedern des National- und 24 Mitgliedern des Ständerates; dabei stimmt die Ratspräsidentin oder der Ratspräsident ausnahmsweise mit (vgl. Art. 80 Abs. 2 ParlG) ${ }^{34}$. Die Zustimmung der Mehrheit der Stimmenden reicht somit unter Umständen nicht aus. ${ }^{35}$ Diese Hürde hat sich bisher allerdings kaum hemmend ausgewirkt. ${ }^{36}$

2. Sachliche und zeitliche Dringlichkeit: Diese Voraussetzung ist nur dann erfüllt, wenn einem rechtspolitisch gewichtigen Anliegen ein nicht wiedergutzumachender Nachteil droht, sollte das Bundesgesetz nicht unverzüglich Geltung erlangen können. ${ }^{37}$ Durch die sofortige Inkraftsetzung dürfte der Zeitgewinn zwischen sechs und zwölf Monaten liegen, ${ }^{38}$ weshalb sich eine Dringlicherklärung und damit der Ausschluss der vorgängigen demokratischen Partizipation nur dann rechtfertigen lassen, wenn ein Gesetz oder eine Gesetzesänderung einen derartigen Aufschub nicht erträgt. ${ }^{39}$ Hier ist also eine Rechtsgüterabwägung vorzunehmen, bei der die Bundesversammlung freilich über einen erheblichen Beurteilungsspielraum verfügt, ${ }^{40}$ an den auch das Bundesgericht gebunden ist. ${ }^{41}$ Klar ist aber, dass allein die Angst vor einem Referendum oder die Absicht, ein bloss nachträgliches Referendum

2003/14. September 2003 [SR 131.226] und § 78 Abs. 4 der Verfassung des Kantons Aargau vom 25. Juni 1980 [SR 131.227]).

$33 \mathrm{Zu}$ denken ist insbesondere an das (suspensive) fakultative Gesetzesreferendum. Ferner kann bei Dringlichkeit die Vernehmlassungsfrist verkürzt werden (vgl. Art. 7 Abs. 4 des Bundesgesetzes vom 18. März 2005 über das Vernehmlassungsverfahren [V1G; SR 172.061]).

34 Bundesgesetz vom 13. Dezember 2002 über die Bundesversammlung (ParlG; SR 171.10).

35 Näheres zum parlamentarischen Verfahren der Dringlicherklärung, das sich nach Art. 77 ParlG richtet, bei Cornelia Theler, in: Martin Graf/Cornelia Theler/Moritz von Wyss (Hrsg.), Parlamentsrecht und Parlamentspraxis der Schweizerischen Bundesversammlung, Kommentar zum Parlamentsgesetz (ParlG) vom 13. Dezember 2002, Basel 2014, Art. 77 N. 1 ff. So gilt etwa das Erfordernis der qualifizierten Mehrheit - anders als bei der Ausgabenbremse (vgl. Art. 159 Abs. 3 Bst. b BV) - auch dann, wenn im (abgekürzten, vgl. Art. 95 Bst. f ParlG) Differenzbereinigungsverfahren der Erstrat ein zweites Mal über die Dringlichkeitsklausel abstimmen muss (vgl. a.a.O., N. 9). Eidgenossenschaft, Zürich 2000, N. 1207; Theler (Fn. 35), Art. 77 N. 12. Vgl. statt vieler SGK-Tschannen (Fn. 4), Art. 165 N. 6. recht, 2. Aufl., Zürich/St.Gallen 2015, § 23 N. 50; Hangartner/KLey (Fn. 36), N. 1200. 
zu ermöglichen, keine Dringlicherklärung begründen kann. ${ }^{42}$ Auch kann die Bundesversammlung, hat sie die Dringlichkeit einmal verneint, indem sie das ordentliche Gesetzgebungsverfahren gewählt hat, nicht ein gleichlautendes Bundesgesetz erlassen und dringlich erklären, wenn gegen die ursprüngliche Vorlage das Referendum ergriffen worden ist. ${ }^{43}$

3. Angemessene Befristung: Dringlich erklärte Bundesgesetze sind zu befristen, wobei sich die Bundesverfassung nicht zur höchstzulässigen Geltungsdauer äussert. Die Befristung sollte wenige Jahre nicht überschreiten; ${ }^{44}$ jedenfalls dürfte eine Befristung von über zehn Jahren kaum mehr verfassungskonform sein. ${ }^{45}$ Dem Verhältnismässigkeitsprinzip entsprechend darf eine Befristung nicht länger dauern, als es die Dringlichkeitslage erfordert; ${ }^{46}$ gleichzeitig gilt es jedoch zu beachten, dass bei «unterjährigen» Bundesgesetzen keine Referendumsmöglichkeit besteht. Mit Ablauf der Befristung tritt das dringlich erklärte Bundesgesetz ohne Weiteres ausser Kraft.

[8] Erfüllt das Bundesgesetz diese Voraussetzungen, so kann es dringlich erklärt und «sofort» (Art. 165 Abs. 1 erster Satz BV), d.h. noch am Tag der Schlussabstimmung, in Kraft gesetzt werden, ${ }^{47}$ wobei es gleichentags auf dem Weg der «dringlichen Veröffentlichung» nach Art. 7 Abs. 3 PublG ${ }^{48}$ zu publizieren ist. Wird das Gesetz noch auf den Tag der Beschlussfassung hin in Kraft gesetzt, so hat dies - wird keine spätere Uhrzeit des betreffenden Tages festgelegt - allerdings eine Rückwirkung zur Folge. Umgekehrt bewirkt die Inkraftsetzung auf einen späteren Zeitpunkt hin unter Umständen eine Verkürzung der Geltungsdauer, da das dringlich erklärte Bundesgesetz allenfalls innert Jahresfrist nach Annahme durch die Bundesversammlung ausser Kraft zu treten hat (vgl. Art. 165 Abs. 2 und 3 BV). ${ }^{49}$

[9] Die direktdemokratischen Mitwirkungsrechte bei dringlichen Bundesgesetzen hängen einerseits von deren Geltungsdauer, andererseits von der Frage ab, ob sich diese auf die Bundesverfassung stützen können oder nicht. «Unterjährige» dringlich erklärte Bundesgesetze sind den Volksrechten vollständig entzogen (vgl. Art. 140 Abs. 1 Bst. c BV e contrario und Art. 141 Bst. b BV e contrario), ${ }^{50}$ was sie als besonders problematisch erscheinen lässt. ${ }^{51}$ Sie dürfen nach Ablauf ihrer Geltungsdauer nicht erneuert werden, ist doch sonst die Gefahr zu gross, dass die Referendums-

42 Vgl. Hangartner/Kley (Fn. 36), N. 1197; Jörg P. Müller, Gebrauch und Missbrauch des Dringlichkeitsrechts nach Artikel 89 ${ }^{\text {bis }}$ BV, Bern 1977, S. 15.

43 Vgl. Hangartner/Kley (Fn. 36), N. 1199.

44 Vgl. Gächter, Staatsrecht (Fn. 38), § 23 N. 52; SGK-Tschannen (Fn. 4), Art. 165 N. 8 spricht davon, dass zwei bis sechs Jahre üblich seien.

45 Vgl. Gä̈hter, Staatsrecht (Fn. 38), § 23 N. 52.

46 Vgl. Hangartner/Kley (Fn. 36), N. 1214.

47 Vgl. Hangartner/Kley (Fn. 36), N. 1215.

48 Bundesgesetz vom 18. Juni 2004 über die Sammlungen des Bundesrechts und das Bundesblatt (PublG; SR 170.512).

49 Vgl. Bundesamt für Justiz (Hrsg.), Gesetzgebungsleitfaden, Leitfaden für die Ausarbeitung von Erlassen des Bundes, 4. Aufl., Bern 2019, N. 1000, 1005. Zudem läuft der Gesetzgeber bei einer Inkraftsetzung auf einen späteren Zeitpunkt hin Gefahr, bei den Betroffenen vorübergehend - d.h. bis zum Tag der Inkraftsetzung - gerade dasjenige Verhalten hervorzurufen, welches mit dem dringlichen Bundesgesetz unterbunden werden sollte.

50 Damit erfüllen sie materiell die Anforderungen des formellen Schweizer Gesetzesbegriffs nicht; aus Art. 165 BV bzw. Art. 140 Abs. 1 Bst. c und Art. 141 Abs. 1 Bst. b BV e contrario geht aber hervor, dass sie als «Bundesgesetze» zu verstehen und zu bezeichnen sind (vgl. dazu Hangartner/KLey [Fn. 36], N. 932, 1191, m.H. auf eine noch andere Begrifflichkeit unter der alten Bundesverfassung).

51 Vgl. GächteR, Demokratie und Dringlichkeit (Fn. 31), S. 94. 
pflicht umgangen wird; ${ }^{52}$ zumindest aber ist zu fordern, dass im Fall einer Verlängerung die gesamte Geltungsdauer für die Frage der Referendumspflicht ausschlaggebend ist. ${ }^{53}$ Die praktische Bedeutung solcher Erlasse und damit einhergehend das demokratische Defizit sind allerdings gering. ${ }^{54}$ Zwischen 1949 und 1999 ergingen 22 «unterjährige» dringliche Bundesbeschlüsse; seit Inkrafttreten der geltenden Bundesverfassung erliess die Bundesversammlung bloss zwei dringliche Bundesgesetze mit einer Geltungsdauer von weniger als einem Jahr. ${ }^{55}$

[10] «Überjährige» dringlich erklärte Bundesgesetze, die sich auf die Bundesverfassung stützen können, ${ }^{56}$ unterliegen einem nachträglichen fakultativen Referendum mit Volksmehr gemäss Art. 141 Abs. 1 Bst. b BV. Da die Referendumsvorlage bereits in Kraft gesetzt wurde, wirkt das Referendum im Unterschied zum «gewöhnlichen» Gesetzesreferendum nicht suspensiv, sondern - im Fall einer Ablehnung des Gesetzes - resolutiv bzw. abrogativ. «Überjährige» dringlich erklärte Bundesgesetze, die keine Verfassungsgrundlage haben, ${ }^{57}$ müssen innerhalb eines Jahres nach ihrem Inkrafttreten von Volk und Ständen angenommen werden; sie unterstehen somit einem nachträglichen obligatorischen Referendum (vgl. Art. 140 Abs. 1 Bst. c BV), das allerdings ein Gesetzesreferendum bleibt. ${ }^{58}$

[11] Wird das Referendum nicht ergriffen bzw. wird das dringlich erklärte Bundesgesetz in einer Volksabstimmung bestätigt, so bleibt es bis zum Ablauf der Geltungsdauer in Kraft, wobei eine dringliche «Erneuerung» (d.h. eine Verlängerung der Befristung) nicht ausgeschlossen ist, sollten die bereits genannten Voraussetzungen (nach wie vor) erfüllt sein (vgl. Art. 165 Abs. 4 BV $e$ contrario), ${ }^{59}$ was allerdings selten der Fall sein dürfte. ${ }^{60}$

[12] Umgekehrt ist eine vorzeitige Ausserkraftsetzung denkbar, sei es durch Bundesversammlung oder Bundesrat aufgrund einer ausdrücklichen Ermächtigung im betreffenden Gesetz, sei es - wenn eine solche fehlt - durch die Bundesversammlung durch Verkürzung der Geltungsdauer, wobei Letzteres - ausser in Fällen praktischer Gegenstandslosigkeit - nur dann als gerechtfertigt erscheint, wenn das dringlich erklärte Bundesgesetz von Volk und Ständen noch nicht angenommen worden ist (obligatorisches Referendum) bzw. die Referendumsfrist noch nicht abgelaufen ist (fakultatives Referendum). ${ }^{61}$ Unzulässig ist insbesondere die Verkürzung der Geltungsdauer aus rein taktischen Gründen, d.h. um das Gesetz der Volksabstimmung zu entziehen, nach-

52 Vgl. Etienne Grisel, Initiative et référendum populaires, Traité de la démocratie semi-directe en droit suisse, 3. Aufl., Bern 2004, N. 857.

53 Vgl. SGK-Tschannen (Fn. 4), Art. 165 BV N. 22; ferner Sebastian Rapp, Direkte Demokratie in der Schweiz, Ein Vergleich zu der Rechtslage in Deutschland, Diss. Marburg, Baden-Baden 2016, S. 378. Vgl. GÄChter, Staatsrecht (Fn. 38), § 23 N. 58.

Vgl. Bundeskanzlei, Übersicht dringliche Bundesbeschlüsse und -gesetze, abrufbar unter https://www.bk.admin. ch/ch/d/pore/vr/vor_2_2_6_5.html.

56 Wann sich ein dringlich erklärtes Bundesgesetz auf die Verfassung stützen kann, d.h. eine Verfassungsgrundlage hat, kann hier nicht weiter ausgeführt werden (vgl. dazu SGK-Tschannen [Fn. 4], Art. 165 BV N. 12 f.).

57 Dringliche Bundesgesetze ohne Verfassungsgrundlage können gemäss herrschender Lehre zwar die verfassungsmässige Zuständigkeitsordnung durchbrechen und damit befristet materielles Verfassungsrecht setzen, müssen aber verfassungsmässige Gebote und Verbote wie etwa das Rechtsgleichheitsgebot oder das Willkürverbot beach ten (vgl. SGK-Tschannen [Fn. 4], Art. 165 N. 18; Judith Wyttenbach, in: Bernhard Waldmann/Eva Maria Belser/ Astrid Epiney [Hrsg.], Bundesverfassung, Basler Kommentar, Basel 2015, Art. 165 N. 17 [zit. BSK-AutoR]; ferner auch Hangartner/KLey [Fn. 36], N. 1192).

Vgl. Hangartner/Kley (Fn. 36), N. 1190.

59 Vgl. statt vieler SGK-Tschannen (Fn. 4), Art. 165 BV N. 22.

60 Vgl. Grisel, Initiative et référendum populaires (Fn. 52), N. 816, 858.

61 Näheres bei Hangartner/KLEy (Fn. 36), N. 352, 1224. 
dem das Referendum bereits ergriffen worden ist. ${ }^{62}$ Wird die obligatorische Abstimmung nicht rechtzeitig durchgeführt oder das dringlich erklärte Gesetz (mit oder ohne Verfassungsgrundlage) in der Abstimmung abgelehnt, so tritt es ein Jahr nach Annahme durch die Bundesversammlung (d.h. nach der Schlussabstimmung) ausser Kraft. Ein negativer Volksentscheid führt also im Bund - anders als in vereinzelten Kantonen $-{ }^{63}$ nicht zwingend dazu, dass ein dringlich erklärtes Bundesgesetz sofort ausser Kraft tritt. Diese ausdrücklich vorgesehene Regelung veranschaulicht schön, wie das nachträgliche, abrogativ wirkende Referendum zwischen den Bedürfnissen der (Dringlichkeits-)Praxis und den Forderungen der direkten Demokratie zu vermitteln versucht. Sprechen praktische Überlegungen nicht dagegen, kann die Bundesversammlung - allenfalls der Bundesrat - das dringliche Bundesgesetz in diesem Fall allerdings auch von sich aus sofort ausser Kraft setzen. ${ }^{64}$ Hingegen ist eine Erneuerung, d.h. eine Verlängerung der ursprünglichen Befristung, nach einer Ablehnung des Gesetzes ausdrücklich ausgeschlossen (vgl. Art. 165 Abs. 4 BV). ${ }^{65}$

[13] Zusammenfassend lässt sich festhalten, dass das Dringlichkeitsrecht zu einer (vorübergehenden) Zurückdrängung der direkten Demokratie als der «Staatsform der Geduld» ${ }^{66}$ führt. Es ermöglicht dem Parlament, "sofort wirkende Fakten [...] mit allen Konsequenzen u.U. für die Rechtsstellung der Einzelnen [zu schaffen].» ${ }^{67}$ Deshalb und insbesondere auch im Hinblick auf die fehlende Verfassungsgerichtsbarkeit gegenüber Bundesgesetzen sollte das Dringlichkeitsrecht von der Bundesversammlung nicht leichtfertig angewendet werden; es soll mithin nur in einer Ausnahmesituation - wie sie die Covid-19-Pandemie darstellt - Anwendung finden. ${ }^{68}$

\section{Dringlichkeitsrecht und Referendum: Praxis}

\subsection{Die Konjunkturen des Dringlichkeitsrechts}

[14] Seit der Einführung des fakultativen bzw. obligatorischen Referendums gegen dringlich erklärte Bundeserlasse machte die Bundesversammlung bis heute insgesamt $149 \mathrm{Mal}$ von der Möglichkeit einer Dringlicherklärung Gebrauch. ${ }^{69}$ Stellt man diese dringlichen Erlasse seit 1949 auf einer Zeitachse dar, so erkennt man deutlich, dass sich über die Jahrzehnte unterschiedliche «Konjunkturen» dringlicher Rechtsetzung abgewechselt haben.

62 Vgl. Hangartner/Kley (Fn. 36), N. 352, 1224; a.M. Bundesrat, Botschaft vom 20. November 1996 über eine neue Bundesverfassung, BBl 1997 I 1 ff., S. 391 und GriseL, Initiative et référendum populaires (Fn. 52), N. 858 («[L]e procédé paraît choquant, mais il découle du système de l'article 165 et n'est donc pas illicite.»); vgl. auch hinten Fn. 74; DERS., a.a.O., N. 814, spricht (im Zusammenhang mit dem obligatorischen Referendum) angesichts des - wenn auch begrenzten - Ermessensspielraums der Bundesversammlung, die Abstimmungspflicht zu «steuern», von einer Einrichtung, die weder ordentliches noch ausserordentliches Referendum sei.

63 Vgl. Autr (Fn. 32), N. 677, 1024, mit Nachweis der einschlägigen Verfassungsbestimmungen der Kantone Zürich, Basel-Stadt, Basel-Landschaft und St. Gallen.

64 Näheres bei Hangartner/KLey (Fn. 36), N. 1221, 1225.

65 Ausführlich zum Erneuerungsverbot z.B. BiagGinI (Fn. 38), Art. 165 BV N. 13.

66 Peter Buss, Das Dringlichkeitsrecht im Bund, Eine Untersuchung über den dringlichen Bundebeschluss nach BV $89^{\text {bis }}$ und die Dringlichkeitspraxis der Bundesversammlung während der Jahre 1960-1980, Diss. Basel 1983, S. 95. BSK-WytTenbach (Fn. 57), Art. 165 N. 12.

68 Vgl. BSK-Wytтenbach (Fn. 57), Art. 165 N. 12.

69 Vgl. Bundeskanzlei, Übersicht dringliche Bundesbeschlüsse und -gesetze, abrufbar unter https://www.bk.admin. ch/ch/d/pore/vr/vor_2_2_6_5.html; Zusammenstellungen und Untersuchungen der Dinglichkeitspraxis der Bundesversammlung nach Art. $89^{\text {bis }}$ aBV vor 1980 finden sich bei Buss (Fn. 66) und Heinz Walter Mäusli, Die dringlichen Bundesbeschlüsse nach Art. 89 $9^{\text {bis }}$ BV, Diss. Zürich 1960. 


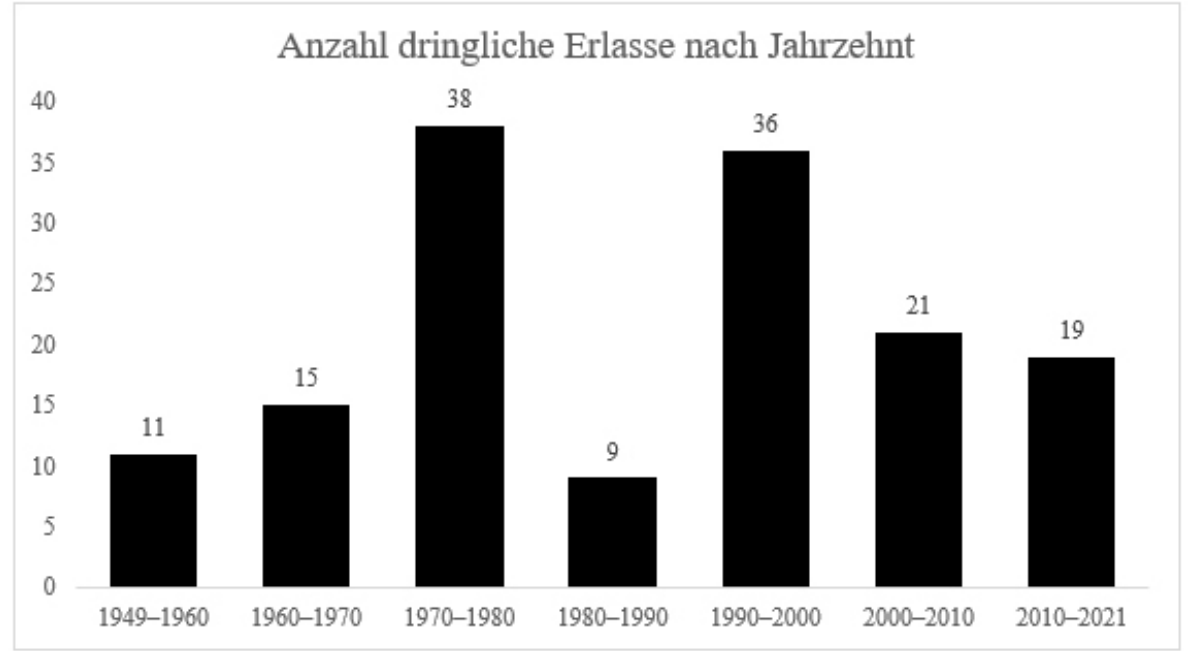

Abbildung: Anzahl dringliche Erlasse nach Jahrzehnt ${ }^{70}$

[15] Die Hochkonjunktur der Wirtschaft in den 1960er- und 1970er-Jahren führte auch zu einer Hochkonjunktur des Dringlichkeitsrechts, und zwar auf den Gebieten der Konjunkturdämpfung, der Land-, insbesondere Milchwirtschaft, der Raumplanung sowie der Währungs-, Geldund Kreditpolitik. Während die Bundesversammlung in den 1980er-Jahren seltener zum Dringlichkeitsrecht griff, setzte mit den 1990er-Jahren erneut eine Phase dringlicher Bundesbeschlüsse ein, wobei diese die verschiedensten Gebiete wie Hypothekarzinsen, Krankenversicherung und Investitionsförderung betrafen. ${ }^{71}$ Auch wenn die beiden ersten Jahrzehnte unter der neuen Bundesverfassung keine mit den 1970er- und 1990er-Jahren vergleichbaren Zahlen hervorbrachten, so musste dennoch wiederholt - am häufigsten in den Bereichen des Sozialversicherungs- und Gesundheitsrechts sowie der Finanz- und Wirtschaftspolitik - auf Dringlichkeitsrecht zurückgegriffen werden. Ohne Corona-Krise sähe das Bild freilich noch ganz anders aus: Alleine seit deren Ausbruch griff die Bundesversammlung zehn Mal zu Dringlichkeitsrecht (Stand 31. Mai 2021), also in rund einem Jahr gleich oft wie im gesamten Jahrzehnt zuvor. Neun Mal handelt es sich um dringliche Bundesgesetze mit Verfassungsgrundlage mit einer Geltungsdauer von mehr als einem Jahr, wobei bisher das fakultative Referendum einzig gegen das Covid-19-Gesetz ergriffen wurde. In einem Fall handelt es sich um eine dringliche Änderung des Parlamentsgesetzes ohne Verfassungsgrundlage, ${ }^{72}$ wobei aufgrund der Geltungsdauer von unter einem Jahr keine obligatorische Abstimmung durchgeführt werden muss.

70 Eigene Darstellung gemäss Bundeskanzlei, Übersicht dringliche Bundesbeschlüsse und -gesetze, abrufbar unter https://www.bk.admin.ch/ch/d/pore/vr/vor_2_2_6_5.html.

71 Vgl. für Beispiele Buss (Fn. 66), S, 203 f.; Grisel, Initiative et référendum populaires (Fn. 52), N. 811, 855; Hangartner/Kley (Fn. 36), N. 1175; Kley, Verfassungsgeschichte (Fn. 15), S. 355, 370 ff.

72 Bundesgesetz vom 13. Dezember 2002 über die Bundesversammlung (ParlG), Änderung vom 10. Dezember 2020 (Covid-19: Teilnahme an Abstimmungen im Nationalrat; Unterbruch oder Verschiebung der Session) (AS 2020 5375). Es ist unter der neuen Bundesverfassung das bisher einzige Beispiel eines dringlichen Bundesgesetzes ohne Verfassungsgrundlage; vgl. dazu Andrea Caroni/Martin Graf, Wahrung der Sessionsteilnahmegarantie in einer Pandemie?, Das dringliche Bundesgesetz ohne Verfassungsgrundlage zur Änderung des Parlamentsgesetzes vom 10. Dezember 2020, in: Jusletter 15. Februar 2021. 


\subsection{Die bisherigen Volksabstimmungen im Überblick}

[16] Bei den zuvor erwähnten 149 dringlichen Erlassen seit 1949 handelte es sich in 13 Fällen (8.7 Prozent) um dringliche Bundesbeschlüsse ohne Verfassungsgrundlage, deren Geltungsdauer ein Jahr überstieg, weshalb sie dem obligatorischen Referendum nach Art. $89^{\text {bis }}$ Abs. 3 aBV unterstanden; durchgeführt wurden aber nur elf Abstimmungen, ${ }^{73}$ wobei Volk und Stände alle Erlasse annahmen.

[17] In weiteren acht Fällen (5.4 Prozent) kam gegen einen dringlichen Bundesbeschluss bzw. ein dringlich erklärtes Bundesgesetz mit Verfassungsgrundlage ein fakultatives Referendum zustande; zur Volksabstimmung kam es aber nur in sieben Fällen. ${ }^{74}$ In diesem Rahmen ist auch die bisher einzige Ablehnung eines dringlichen Bundeserlasses durch die Stimmberechtigten zu verzeichnen. So verwarfen sie den dringlichen Bundesbeschluss über die Finanzierung der Arbeitslosenversicherung ${ }^{75}$ in der Volksabstimmung vom 28. September 1997 knapp mit einem Nein-Stimmen-Anteil von 50.8 Prozent, worauf dieser per 1. Dezember 1997 ausser Kraft trat. ${ }^{76}$ Kantonale Arbeitslosenkomitees und Gewerkschaften aus der Westschweiz hatten damals gegen die vorgesehenen Kürzungen bei der Arbeitslosenversicherung erfolgreich das Referendum ergriffen und waren schliesslich - dank überdurchschnittlich starker Unterstützung aus der Westschweiz sowie von Frauen und Jungen - siegreich. Dabei profitierte das Referendumskomitee in den Wochen vor dem Urnengang auch «von unsensiblen Äusserungen» eines Vizedirektors des zuständigen Bundesamts gegenüber Arbeitslosen und vom Bekanntwerden weiterer, von der Bundesverwaltung erwogener «massive[r] Leistungskürzungen bei der ALV». ${ }^{77}$

[18] Da die Stimmenden aber alle anderen Referendumsvorlagen guthiessen, steht heute eine einzige Ablehnung eines dringlichen Bundeserlasses insgesamt 17 Annahmen gegenüber, was gerade einmal einer Ablehnungsquote von 5.6 Prozent entspricht. Gemessen an der bei 35.7 Prozent liegenden Gesamtquote aller abgelehnten fakultativen Referendumsvorlagen seit Einführung des nachträglichen Referendums 1949, ${ }^{78}$ ist dies eine auffällig tiefe Quote. Die Vermutung liegt deshalb nahe, dass sich die Willensbildung der Stimmberechtigten im Fall eines nachträglichen, höchstens abrogativ wirkenden Referendums von derjenigen im Fall eines suspensiv wirkenden Referendums unterscheidet. ${ }^{79}$ Diese Frage kann hier zwar nicht weiter vertieft werden, doch ist immerhin anzumerken, dass ein bereits geltendes Gesetz allenfalls schon vor der Volksabstim-

73 Der Bundesbeschluss vom 21. Dezember 1949 betreffend die Übergangsordnung des Finanzhaushaltes des Bundes (Finanzordnung 1950 und 1951; AS 1949 1801) wurde im Jahr 1950 noch vor Ablauf der Jahresfrist durch einen ordentlichen Beschluss ersetzt (AS 1950 1463). Der Bundesbeschluss vom 7. Oktober 1977 über den Schutz der Währung (AS 1977 1859) wurde im Sommer 1978 nachträglich durch einen Beschluss ersetzt, der sich auf den neuen Konjunkturartikel in der Bundesverfassung stützte und damit eine Verfassungsgrundlage hatte (AS 1978 1436).

74 Gegen den Bundesbeschluss vom 31. Januar 1975 über die Festsetzung des Beitrages des Bundes an die Alters- und Hinterlassenenversicherung (AS 1975 181) kam zwar das Referendum zustande, doch wurde dieser noch vor der Abstimmung vom 12. Juni 1975 durch einen ordentlichen Bundesbeschluss ersetzt, gegen den das Referendum nicht ergriffen wurde (AS 1975 1805).

Vgl. Roswitha Dubach, Souverän lehnt weitere Kürzungen bei der Arbeitslosenversicherung hauchdünn ab, in: Wolf Linder/Christian Bolliger/Yvan Rielle (Hrsg.), Handbuch der eidgenössischen Volksabstimmungen 1848-2007, Bern 2010, S. 556.

77 Dubach (Fn. 76), S. 556.

7851 Ablehnungen stehen in diesem Zeitraum 93 Annahmen gegenüber; eigene Berechnung anhand Bundeskanzlei, Zustandegekommene Referenden, abrufbar unter https://www.bk.admin.ch/ch/d/pore/rf/ref_2_2_3_2.html. 
mung Gelegenheit hatte, sich zu bewähren, ${ }^{80}$ gleichzeitig aber auch Fakten schaffen kann, welche die Stimmberechtigten in eine gewisse Zwangslage versetzen; jedenfalls ist «[d]ie Neigung des Volkes, vollendeten Tatsachen zuzustimmen, [... ein bekanntes Phänomen». ${ }^{81}$ Überdies könnte es leichter fallen, einen Erlass anzunehmen, dessen provisorischer Charakter aufgrund der Befristung von vornherein bekannt ist. ${ }^{82}$

[19] Das Phänomen der Status-quo-Verzerrung dürfte sich, wenn man die hohen Zustimmungsraten beachtet, auch bei den bisherigen Abstimmungen in diesem Jahrtausend ausgewirkt haben, wobei seit dem Inkrafttreten der Bundesverfassung von 1999 bis zum aktuellen Fall des Covid-19-Gesetzes erst zwei dringlich erklärte Bundesgesetze zur Volksabstimmung kamen. So nahm das Volk am 9. Februar 2003 mit 77.4 Prozent Ja-Stimmen-Anteil - allerdings bei einer sehr tiefen Stimmbeteiligung von knapp 29 Prozent - überaus deutlich das dringlich erklärte Bundesgesetz über die Anpassung der kantonalen Beiträge für die innerkantonalen stationären Behandlungen nach dem Bundesgesetz über die Krankenversicherung ${ }^{83}$ an. ${ }^{84}$ Dabei handelte es sich allerdings um eine blosse Übergangsregelung, die - als Folge eines Entscheids des Eidgenössischen Versicherungsgerichts - die Kostentragung von stationären Behandlungen zwischen Kantonen und Krankenversicherern für einen Zeitraum von zwei Jahren neu festlegte; dies vor dem Hintergrund, dass danach ohnehin eine Totalrevision des Bundesgesetzes über die Krankenversicherung ${ }^{85}$ geplant war. ${ }^{86}$ Dem vom Krankenversicherer Assura ergriffenen Referendum wurde von Anfang an keine Chance eingeräumt, weshalb es auch keinen eigentlichen Abstimmungskampf gab. ${ }^{87}$ Mit 78.4 Prozent gar eine noch höhere Zustimmung ${ }^{88}$ erfuhr am 9. Juni 2013 die dringliche Änderung des Asylgesetzes ${ }^{89}$, diesmal immerhin bei einer Stimmbeteiligung von knapp 40 Prozent. Aufgrund einer hohen Zahl an Asylgesuchen zu Beginn der 2010er-Jahre ergriffen Bundesversammlung und Bundesrat mittels Dringlichkeitsrecht Massnahmen, um das Asylsystem zu entlasten, ${ }^{90}$ wobei aber angedacht war, diese ohnehin nachfolgend in das ordentliche Recht zu überführen. ${ }^{91}$ Dagegen ergriffen die Jungen Grünen gemeinsam mit einigen asylund migrationspolitischen Organisationen das Referendum.

80 Vgl. Haller/Kölz/Gächter (Fn. 5), N. 272.

81 Hangartner/Kley (Fn. 36), N. 1176.

82 Vgl. Grisel, Initiative et référendum populaires (Fn. 52), N. 811.

83 Bundesgesetz vom 21. Juni 2002 über die Anpassung der kantonalen Beiträge für die innerkantonalen stationären Behandlungen nach dem Bundesgesetz über die Krankenversicherung (AS 2002 1643).

84 Vgl. Bundesratsbeschluss vom 25. März 2003 über das Ergebnis der Volksabstimmung vom 9. Februar 2003 (BBl 2003 3111).

85 Bundesgesetz vom 18. März 1994 über die Krankenversicherung (KVG; SR 832.10).

Vgl. Erläuterungen des Bundesrates zur Volksabstimmung vom 9. Februar 2003, S. 11.

87 Vgl. Roswitha Dubach, Kantone müssen die Spitalkosten für Zusatzversicherte nur stufenweise übernehmen, in: Linder/Bolliger/Rielle (Fn. 76), S. 627 f.

88 Vgl. Bundesratsbeschluss vom 26. Juli 2013 über das Ergebnis der Volksabstimmung vom 9. Juni 2013 (BBl 2013 6613).

89 Asylgesetz vom 26 Juni 1998 (AsylG), Änderung vom 28. September 2012 (Dringliche Änderungen des Asylgesetzes) (AS 20125359 ).

90 Vgl. Zusatzbotschaft vom 23. September 2011 zur Änderung des Asylgesetzes (Kurzfristige Massnahmen), BBl 20117325.

91 Vgl. Erläuterungen des Bundesrates zur Volksabstimmung vom 9. Juni 2013, S. 20; ferner grafische Darstellung des EJPD, abrufbar unter https://www.ejpd.admin.ch/dam/sem/de/data/aktuell/news/2013/2013-03-25/ revision-asylg-d.pdf.download.pdf/revision-asylg-d.pdf. 
[20] Diese Abstimmungen unterscheiden sich allerdings insofern vom Referendum zum Covid19-Gesetz, als es bisher jeweils nur um eine einzige, klar abgrenzbare Abstimmungsmaterie ging, weshalb eine Ablehnung auch eine überschaubare Wirkung gehabt hätte. Beim Covid-19-Gesetz geht es demgegenüber nicht um die punktuelle Bekämpfung eines dringlichen Einzelproblems, vielmehr stellt es das rechtliche Fundament für die generelle Bewältigung eines Grossteils der gegenwärtigen Krise dar. Inwiefern sich dies auf die Zustimmung auswirken wird, bleibt abzuwarten. Abstimmungsumfragen deuten jedenfalls auch beim Covid-19-Gesetz auf eine hohe Zustimmung hin. ${ }^{92}$

\section{Probleme des Referendums gegen das Covid-19-Gesetz}

\subsection{Die Ausgangslage}

[21] In der Herbstsession 2020 befasste sich die Bundesversammlung erstmals intensiv mit dem neuen Covid-19-Gesetz. Mit der Vorlage sollte, wo notwendig, eine gesetzliche Grundlage für den Inhalt der Corona-Notverordnungen, die der Bundesrat seit dem Frühjahr 2020 erlassen hatte, geschaffen werden, um die beschlossenen Massnahmen weiterhin aufrechterhalten zu können. ${ }^{93}$ Auch nach zwei Beratungsrunden waren sich der Nationalrat und der Ständerat noch nicht in allen Punkten einig, wie das Covid-19-Gesetz aussehen sollte. Erst die Einigungskonferenz brachte den endgültigen Durchbruch.

[22] Das Covid-19-Gesetz regelt gemäss dem in «schönstem» Substantivstil gehaltenen Art. 1 Abs. 1 «besondere Befugnisse des Bundesrates zur Bekämpfung der Covid-19-Epidemie und zur Bewältigung der Auswirkungen der Bekämpfungsmassnahmen auf Gesellschaft, Wirtschaft und Behörden». Dabei reichen die geregelten Materien, um nur ein paar Beispiele zu nennen, von umfangreichen finanziellen Unterstützungsmassnahmen über Massnahmen im Ausländer- und Asylbereich bis hin zu Massnahmen der Gesundheitsversorgung, wobei mit Art. 18 sogar eine Strafbestimmung Eingang in das Gesetz fand.

[23] Zwar regelt das Gesetz die erwähnten besonderen Befugnisse des Bundesrates. Dennoch stellt sich die Frage, ob es den Grundsatz der Einheit der Materie wahrt, ${ }^{94}$ wobei offenbleiben kann, ob es sich dabei um eine rechtliche Verpflichtung oder ein blosses «Zweckmässigkeitskriterium» für den Bundesgesetzgeber handelt. ${ }^{95}$ Jedenfalls wurde durch die Schaffung eines solchen «Sammelgefässes» ${ }^{96}$ die Zwangslage, die bei dringlichen Bundesgesetzen ohnehin tendenziell schon besteht (sowohl für Parlamentarierinnen und Parlamentarier, die rasch handeln müssen, als auch für Stimmberechtigte, die sich mit vollendeten Tatsachen konfrontiert sehen)

92 Fast alle etablierten Parteien beschlossen die Ja-Parole; einzig die SVP beschloss Stimmfreigabe. Eine klare Mehrheit von 67 Prozent der Teilnahmewilligen hätten Ende April für das Covid-19-Gesetz gestimmt, vgl. Forschungsinstitut gfs. Bern, Erste Welle der SRG-SSR-Trendbefragung zu den Volksabstimmungen vom 13. Juni 2021, realisiert zwischen dem 19. April und dem 2. Mai 2021 bei 22'732 Stimmberechtigten, abrufbar unter https://cockpit.gfsbern.ch/de/cockpit/srg_trend_032021-3/.

93 Vgl. Art. $7 d$ Abs. 2 Regierungs- und Verwaltungsorganisationsgesetz vom 21. März 1997 (RVOG; SR 172.010).

94 Die Botschaft vom 12. August 2020 zum Bundesgesetz über die gesetzlichen Grundlagen für Verordnungen des Bundesrates zur Bewältigung der Covid-19-Epidemie (Covid-19-Gesetz), BBl 2020 6563, S. 6621, bejaht die Wahrung des Grundsatzes der Einheit der Materie mit knapper Begründung.

95 Dazu jüngst und m.w.H. Andrea CAroni, Gilt die Einheit der Materie auch für die Bundesgesetzgebung?, in: Parlament, Parlement, Parlamento 1/21, S. 56 ff., insbes. S. 61.

96

Botschaft zum Covid-19-Gesetz (Fn. 94), S. 6574. 
noch erheblich vergrössert. Nicht völlig befriedigend wäre allerdings auch eine Aufteilung der Vorlage in mehrere dringliche Bundesgesetze gewesen: ${ }^{97}$ Einerseits drängte wegen der sich rasch ändernden epidemiologischen Situation die Zeit, andererseits hätten die Gegner des Covid-19Gesetzes unter Umständen gegen zahlreiche Gesetze das Referendum ergreifen müssen, was in Pandemiezeiten - das Sammeln von Unterschriften ist aufgrund der zu berücksichtigenden Hygienemassnahmen und fehlender Veranstaltungen momentan eher aufwendig - schwieriger zu bewältigen gewesen wäre.

[24] Darüber hinaus strapaziert - das sei hier am Rande vermerkt - das Covid-19-Gesetz mit seinen zahlreichen Delegations- bzw. Ermächtigungsnormen auch den materiellen Gesetzesbegriff. So ist zweifelhaft, ob es mit Blick auf die Forderung, dass «[a]lle wichtigen rechtsetzenden Bestimmungen [...] in der Form des Bundesgesetzes zu erlassen» sind (Art. 164 Abs. 1 BV), die jeweils an erster Stelle genannte Voraussetzung der Gesetzesdelegation einhält, wonach Rechtsetzungsbefugnisse durch Bundesgesetz nur übertragen werden können «soweit dies nicht durch die Bundesverfassung ausgeschlossen wird» (Art. 164 Abs. 2 BV). ${ }^{98}$

[25] Wie einleitend bereits erwähnt, trat das von der Bundesversammlung für dringlich erklärte Covid-19-Gesetz vom 25. September 2020 bereits am folgenden Tag in Kraft. Für das neue Gesetz stimmten in der Schlussabstimmung insgesamt 153 (78.5 Prozent) Nationalrätinnen und Nationalräte, dagegen lediglich 36 (aus der SVP-Fraktion) bei 6 Enthaltungen. ${ }^{99}$ Das nötige Quorum im Nationalrat (mindestens 101 Stimmen) für die Dringlicherklärung war mit 165 Stimmen schon zuvor ebenfalls mit Leichtigkeit erreicht worden. ${ }^{100}$ In der "chambre de réflexion» wurde das Gesetz ohne Gegenstimme angenommen und für dringlich erklärt. ${ }^{101}$ Das Gesetz stützt sich gemäss Ingress auf zahlreiche Artikel der Bundesverfassung, womit die Bundesversammlung von einem dringlichen Bundesgesetz mit Verfassungsgrundlage ausgeht. Die Geltungsdauer des Bundesgesetzes wurde ursprünglich grundsätzlich bis zum 31. Dezember 2021 befristet, wobei zwei Artikel länger, konkret bis zum 31. Dezember 2022, ${ }^{102}$ und ein Artikel lediglich bis zum 30. Juni 2021 in Kraft bleiben sollen. ${ }^{103}$ Die erste Fassung des Covid-19-Gesetzes wurde aber insgesamt als überjähriges dringliches Bundesgesetz mit Verfassungsgrundlage behandelt, weshalb es einem nachträglichen fakultativen Referendum unterstellt wurde. ${ }^{104}$

[26] Aufgrund der sich ständig ändernden Pandemie-Situation musste die Bundesversammlung das Gesetz bereits zweimal erheblich revidieren, wobei insgesamt 31 Artikel geändert bzw. ergänzt wurden. ${ }^{105}$ Dies ist insofern beachtlich, als das ursprüngliche Gesetz bloss 21 Artikel umfasste. Eine erste gewichtige Revision nahm die Bundesversammlung am 18. Dezember 2020 vor, als sie insgesamt - unter Einschluss des Ingresses - 10 Artikel änderte bzw. neu einfügte und die

97 Gemäss Botschaft zum Covid-19-Gesetz (Fn. 94), S. 6574, ging der Vernehmlassungsvorlage «eine Aussprache im Bundesrat voraus, in der alternative Modelle - Mantelerlass mit Änderungen in bestehenden Gesetzen, mehrere Einzelvorlagen - diskutiert und schliesslich verworfen wurden».

Vgl. auch GÄCHTER, Staatsrecht (Fn. 38), § 22 N. 35.

Vgl. AB 2020 N 1962; für das Abstimmungsverhalten der Nationalräte, vgl. https://www.parlament.ch/poly/ Abstimmung/51/out/vote_51_21547.pdf.

100 Vgl. AB 2020 N 1819.

101 Vgl. AB 2020 S 1024, 1075.

102 Vgl. Art. 1 und 17 Bst. a-c Covid-19-Gesetz (Stand 26. September 2020).

103 Vgl. Art. 15 Covid-19-Gesetz (Stand 26. September 2020).

104 Bei Art. 15 des Covid-19-Gesetzes handelt es sich um eine «unterjährige» dringliche Regelung, die grundsätzlich dem Referendum nicht zugänglich wäre.

105 Vgl. AS 2020 5821; AS 2021153. 
Befristung vereinzelter Artikel verlängerte. Im gleichen Änderungserlass modifizierte sie überdies das Ordnungsbussengesetz ${ }^{106}$ und das Bundesgesetz über Überbrückungsleistungen für ältere Arbeitslose ${ }^{107}$. In der Schlussabstimmung stimmten im Nationalrat knapp 95 Prozent für die Änderungen; einzig sieben Mitglieder der SVP-Fraktion stimmten dagegen. ${ }^{108}$ Im Ständerat erreichte man erneut Einstimmigkeit. ${ }^{109}$ Auch das qualifizierte Mehr für die Dringlicherklärung war in beiden Räten zuvor wiederum problemlos erreicht worden. Ein zweites Änderungspaket beschloss die Bundesversammlung am 19. März 2021, als sie insgesamt 21 Artikel änderte bzw. neu einfügte. Mit demselben Beschluss änderte sie zudem das Arbeitslosenversicherungsgesetz ${ }^{110}$ und wiederum das Bundesgesetz über Überbrückungsleistungen für ältere Arbeitslose. Was die Befristungen angeht, so sind nun für zahlreiche Artikel unterschiedliche Geltungsdauern vorgesehen, wobei einige Artikel gar bis zum 31. Dezember 2031 in Kraft bleiben sollen. Auch bei diesen Änderungen war man sich im Nationalrat durchgehend einig; bei einem Ja-StimmenAnteil von knapp 87 Prozent waren es wiederum nur einzelne Mitglieder der SVP-Fraktion (13), die dem Revisionspaket in der Schlussabstimmung nicht zustimmten. ${ }^{111}$ Im Ständerat fiel das Resultat wiederum einstimmig aus. ${ }^{112}$ Auch die Dringlicherklärung war in beiden Räten unbestritten.

[27] Erwähnenswert ist zudem, dass die beiden Revisionsbeschlüsse vom 18. Dezember 2020 und 19. März 2021 je gesondert dem (nachträglichen) Referendum unterstellt wurden, dieses jedoch (noch) nicht ergriffen wurde. ${ }^{113}$

\subsection{Der Gegenstand der Volksabstimmung}

[28] Angesichts dieser Ausgangslage fällt es schwer, sich nur schon Klarheit über den genauen Abstimmungsgegenstand vom kommenden 13. Juni zu verschaffen: Einerseits weisen verschiedene Artikel unterschiedliche Geltungsdauern auf, anderseits führt eine - soweit ersichtlich im Dringlichkeitsrecht erstmals auftretende - Kuriosität zu einer weiteren Verkomplizierung dieses «eigenartigsten Referendum[s] der Schweizer Geschichte» ${ }^{114}$ : Zum Zeitpunkt der Volksabstimmung am 13. Juni 2021 wird das Covid-19-Gesetz aufgrund der sich stetig ändernden PandemieSituation bereits zwei Mal erheblich revidiert worden sein. Die Unterschriftensammlung des Referendumskomitees erfolgte jedoch im Zeitraum zwischen Oktober 2020 und Januar 2021, also

106 Ordnungsbussengesetz vom 18. März 2016 (OBG; SR 314.1).

107 Bundesgesetz vom 19. Juni 2020 über Überbrückungsleistungen für ältere Arbeitslose (ÜLG; BBl 2020 5519, noch nicht in Kraft).

108 Vgl. AB 2020 N 2371, für das Abstimmungsverhalten der Nationalräte, vgl. https://www.parlament.ch/poly/ Abstimmung/51/out/vote_51_22199.pdf.

109 Vgl. AB 2020 S 1440

110 Bundesgesetz vom 25. Juni 1982 über die obligatorische Arbeitslosenversicherung und die Insolvenzentschädigung (AVIG; SR 837.0).

111 Vgl. AB 2021 N 717, für das Abstimmungsverhalten der Nationalräte, vgl. https://www.parlament.ch/poly/ Abstimmung/51/out/vote_51_22767.pdf.

112 Vgl. AB 2021 S 342.

113 Vgl. Bundeskanzlei, Vorlagen mit laufender Referendumsfrist, abrufbar unter https://www.bk.admin.ch/ch/d/ pore/rf/ref_1_3_2_1.html.

114 Fabian Fellmann, Das eigenartigste Referendum der Schweizer Geschichte, Tages-Anzeiger online, 6. Mai 2021, abrufbar unter https://www.tagesanzeiger.ch/das-eigenartigste-referendum-der-schweizer-geschichte518856126089. 
in einer Phase, als noch die ursprüngliche Fassung des Gesetzes in Kraft war und die Bundesversammlung erst über eine erstmalige Gesetzesrevision beriet. Es stellt sich somit die Frage, worüber bzw. über welche Fassung des Gesetzes am 13. Juni 2021 wirklich abgestimmt wird: über die ursprüngliche Fassung vom 25. September 2020, über diejenige, welche im Zeitpunkt der Referendumseinreichung am 12. Januar 2021 in Kraft war, oder doch über diejenige, welche gegenwärtig gilt?

[29] Da das Referendumskomitee das Referendum gegen die ursprüngliche Fassung vom 25. September 2020 ergriffen hatte und der Grossteil der Unterschriften zu einem Zeitpunkt gesammelt wurde, in dem diese erste Fassung in Kraft war, spricht aus rein rechtlicher Sicht vieles dafür, dass Gegenstand der Volksabstimmung nun auch diese erste Fassung ist. Nur so lässt sich nämlich für die Unterzeichnenden die nötige Transparenz herstellen, die sich als verfassungsmässiges Recht aus Art. 34 Abs. 2 BV ableiten lässt, da die Garantie der politischen Rechte mitunter auch die freie und unverfälschte Willensbildung der Unterzeichnenden von Volksbegehren schützt. Unter dem Gesichtspunkt dieses verfassungsmässigen Rechts erschiene es demnach als problematisch, wenn in der Volksabstimmung nun eine Fassung zur Diskussion stünde, die zum Zeitpunkt der Unterschriftensammlung noch gar nicht existiert hatte. Wer garantiert nämlich, dass die Unterzeichnenden des Referendums dieses auch unterstützt hätten, wenn nicht die damals geltenden, sondern die spätere, stark revidierte Fassung zur Diskussion gestanden hätte? Auch dass beide Revisionsbeschlüsse jeweils einem eigenen nachträglichen Referendum unterstanden bzw. unterstehen, spricht für eine solche Vorgehensweise. Tatsächlich ist im «Abstimmungsbüchlein» als Abstimmungstext auch die ursprüngliche Fassung des Covid-19Gesetzes abgedruckt. ${ }^{115}$ Formeller Abstimmungsgegenstand ist somit das Covid-19-Gesetz vom 25. September 2020 (Stand 26. September 2020), obwohl es sich dabei um eine Version des Erlasses handelt, die längst nicht mehr in Kraft ist.

\subsection{Die Wirkungen einer allfälligen Ablehnung des Gesetzes}

\subsubsection{Schicksal des Covid-19-Gesetzes}

[30] Was aber geschieht nun, falls das Gesetz an der Urne abgelehnt wird? Im "Abstimmungsbüchlein» wird diese Frage wie folgt beantwortet: «Wenn die Stimmbevölkerung das Covid-19Gesetz ablehnt, tritt es grundsätzlich ein Jahr nach der Annahme durch das Parlament ausser Kraft, also am 25. September 2021.»116 Was bei einer Ablehnung mit den geänderten bzw. neu eingefügten Bestimmungen geschieht, die nicht Gegenstand der Volksabstimmung sind, wird dagegen nicht spezifisch erläutert. Dies deutet daraufhin, dass bei einem negativen Volksentscheid das gesamte Covid-19-Gesetz am 25. September 2021 ausser Kraft treten wird. In diesem Sinne beantwortete der Bundesrat auch eine Frage von Nationalrat Beat Flach nach dem Schicksal der Änderungen des Covid-19-Gesetzes. Der Bundesrat führte aus, dass im Fall einer Ablehnung des Gesetzes in der Volksabstimmung «[a]lle Änderungen des Covid-19-Gesetzes, welche die Bundesversammlung seit dem 25. September 2020 beschlossen und dringlich in Kraft gesetzt

115 Vgl. Erläuterungen des Bundesrates zur Volksabstimmung vom 13. Juni 2021, S. 46 ff.

116 Erläuterungen des Bundesrates zur Volksabstimmung vom 13. Juni 2021, S. 41. 
hat, $[\ldots]{ }^{117}$ dahinfallen würden. ${ }^{118}$ Die vom Referendum nicht erfassten Bestimmungen dürften dann allerdings nicht ohne Weiteres ausser Kraft treten, sondern wären wohl formell noch aufzuheben.

[31] Diese Vorgehensweise ist zwar aus verfassungsrechtlicher Sicht keineswegs zwingend, wohl aber als einzige praktikabel. Denn was geschähe etwa mit den Artikeln, die in der Zwischenzeit abgeändert worden sind? Sind diese nun «immunisiert», da sie nicht Gegenstand der Abstimmung sind, sondern einem eigenen nachträglichen Referendum unterstanden? Als Beispiel kann Art. 2 Abs. 1 genannt werden, der Massnahmen im Bereich der politischen Rechte vorsieht. Der Wortlaut von Abs. 1, der zur Abstimmung gelangt, lautet: «Der Bundesrat kann zur Unterstützung der Ausübung der politischen Rechte vorsehen, dass Referendumsbegehren mit der nötigen Anzahl Unterschriften, jedoch auch ohne Stimmrechtsbescheinigung innerhalb der Referendumsfrist bei der Bundeskanzlei einzureichen sind.» Im März 2021 wurde die Bestimmung dahingehend ergänzt, dass diese Erleichterung nun auch für Initiativbegehren gelten soll. Diese Änderung unterstand gemeinsam mit den übrigen Änderungen vom März 2021 einem eigenen nachträglichen Referendum. Hat dies nun zur Folge, dass Art. 2 Abs. 1 durch diese Änderung in seiner Gesamtheit «immunisiert» wurde? Oder würde ein negativer Urnenentscheid bewirken, dass die Erleichterung für Referenden wegfallen, für Initiativen aber beibehalten würde? Da eine solche Lösung als wenig sinnvoll, ja sogar willkürlich erschiene, ist es konsequent, bei einer Ablehnung des ursprünglichen Wortlauts der Bestimmung auch eine gesamthafte Ablehnung der neuformulierten Bestimmung anzunehmen.

[32] Weniger zwingend erscheint dies jedoch bei einigen neu eingefügten Artikeln, die auch ohne ihr «Grundgerüst» durchaus weiterbestehen könnten. So wurde etwa im März 2021 ein Art. 3a in das Gesetz eingefügt, wonach Personen, die mit einem zugelassenen Impfstoff geimpft sind, keine Quarantäne auferlegt werden darf. Dieser Artikel könnte ohne Weiteres ohne den Art. 3, der (weitere) Massnahmen im Bereich der Gesundheitsversorgung regelt, bestehen. Man müsste also für alle vom Referendum nicht tangierten Bestimmungen gesondert prüfen, ob sie auch nach dem 25. September 2021 in Kraft bleiben könnten. Auch eine solche, der Rechtssicherheit nicht zuträgliche Situation wäre gerade in unsicheren Pandemiezeiten unerwünscht.

\subsubsection{Schicksal der wirtschaftlichen Unterstützungsmassnahmen}

[33] Besonders weitreichende Folgen hätte eine Ablehnung für weite Teile der Wirtschaft, da auch die wirtschaftlichen Unterstützungsmassnahmen zur Bewältigung der Corona-Krise bei einem negativen Volksentscheid ab dem 25. September 2021 über keine gesetzliche Grundlage mehr verfügen würden. Bundesrat und Bundesversammlung müssten hierfür von neuem eine gesetzliche Grundlage schaffen. Von einer allfälligen Ablehnung nicht betroffen wären allerdings Massnahmen des Bundesrates zur Bekämpfung der Pandemie wie etwa Veranstaltungsverbote,

117 Fragestunde, Frage Flach Beat (21.7291), AB 2021 N 443 «Referendumsabstimmung zum Covid-19-Gesetz», abrufbar unter https://www.parlament.ch/de/ratsbetrieb/suche-curia-vista/geschaeft?AffairId=20217291.

118 Vgl. Stellungnahme des Bundesrates vom 26. Mai 2021 zur Interpellation Addor Jean-Luc (21.3588), «Wurden die Schweizerinnen und Schweizer mit den Abstimmungserläuterungen über das Covid-19-Gesetz getäuscht?», abrufbar unter https://www.parlament.ch/de/ratsbetrieb/suche-curia-vista/geschaeft?AffairId=20213588; ferner auch Rubrik «Häufige Fragen und Antworten» zum Covid-19-Gesetz auf der Internetseite des Eidgenössischen Departements des Innern, abrufbar unter https://www.edi.admin.ch/edi/de/home/dokumentation/abstimmungen/ covid-19-gesetz.html. 
die sich auf das Epidemiengesetz ${ }^{119}$ stützen. Wie bereits erwähnt, darf ein dringlich erklärtes Bundesgesetz, das in der Volksabstimmung abgelehnt wird, gemäss ausdrücklichem Wortlaut von Art. 165 Abs. 4 BV nicht erneuert werden, mithin darf der verworfene Erlass nicht sofort wieder im Dringlichkeitsverfahren neu beschlossen werden. ${ }^{120}$ Andernfalls würde das Abstimmungsergebnis ignoriert, weshalb es auch unzulässig ist, inhaltlich fast identische Regelungen erneut dringlich zu erlassen. ${ }^{121}$ Im Vorfeld der Abstimmung wurden nun Überlegungen angestellt, zumindest die «unbestrittenen» Teile des Covid-19-Gesetzes, mithin die Wirtschaftshilfen, in neues Dringlichkeitsrecht «zu giessen». ${ }^{122}$ Eine solche Vorgehensweise wäre verfassungswidrig. Im Übrigen lassen sich die «unbestrittenen» Teile - soweit überhaupt Konsens darüber besteht, wann sie als «unbestritten» gelten können ${ }^{123}$ - nicht eruieren, da die Stimmenden ihr Abstimmungsverhalten bekanntlich nicht begründen müssen bzw. können. Verfassungsrechtlich fragwürdig wäre es deshalb, bei einem ablehnenden Volksentscheid die wirtschaftlichen Unterstützungsmassnahmen als «unbestritten» zu qualifizieren und diese in ein eigenes dringliches Bundesgesetz zu überführen. Der Gesetzgeber ist mit der Vermengung von wirtschaftlichen Unterstützungsmassnahmen und umstritteneren Themen wie etwa Massnahmen im Bereich der Gesundheitsversorgung in einem einzigen Bundesgesetz das Risiko einer Gesamtablehnung bewusst eingegangen. ${ }^{124}$ Ein solches Risiko liesse sich nur vermindern, wenn mehrere Einzelvorlagen beschlossen würden, ${ }^{125}$ da im Bund - anders als nach dem Verfassungsrecht einiger Kantone - ${ }^{126}$ ein Referendum nur gegen einzelne Teile einer Gesamtvorlage («Teilreferendum») nicht vorgesehen ist.

[34] Dem Gesetzgeber bliebe also nur der Weg über das (aufwendige) ordentliche Gesetzgebungsverfahren, wobei die Referendumsfrist von 100 Tagen abgewartet werden müsste. Selbst wenn die Bundesversammlung in der Herbstsession 2021 ein allfälliges Nachfolgegesetz beschliessen könnte, würde bis zu dessen Inkrafttreten eine Lücke von einigen Monaten entstehen ${ }^{127}$ - ein angesichts der Umfrageergebnisse allerdings eher unwahrscheinliches Szenario. ${ }^{128}$

119 Bundesgesetz vom 28. September 2012 über die Bekämpfung übertragbarer Krankheiten des Menschen (EpG; SR 818.101).

120 Vgl. vorne 2.2.

121 Vgl. Jean-François Aubert, in: Jean-François Aubert/Pascal Mahon, Petit commentaire de la Constitution fédérale de la Confédération suisse du 18 avril 1999, Zürich/Basel/Genf 2003, Art. 165 N. 9. In der Literatur wird verschiedentlich eine Erneuerung in einem dringlichen Bundesgesetz dann für zulässig erachtet, wenn sich die Situation «in der Zwischenzeit offensichtlich und wesentlich verändert [habe]» (BSK-WytтenBACH [Fn. 57], Art. 165 N. 22; ähnlich auch Hangartner/KLey [Fn. 36], N. 1223).

122 Vgl. Hansueli SснӧсндI, Die Corona-Nothilfen sind infrage gestellt, NZZ vom 5. Mai 2021, S. 21.

123 Reicht für die «Unbestrittenheit» etwa eine hypothetische Zustimmung von 51 Prozent?

124 Umgekehrt kann der Gesetzgeber von einer Vermischung auch profitieren, sollten viele Stimmberechtigte das gesamte Covid-19-Gesetz aufgrund der wichtigen Unterstützungsmassnahmen trotz Zweifel als nicht «ablehnbar» ansehen.

125 Vgl. dazu vorn Fn. 97.

126 Vgl. Art. 34 Abs. 1 Bst. b der Verfassung des Kantons Zürich vom 27. Februar 2005 (SR 131.211); § 34 Abs. 6 der Verfassung des Kantons Zug vom 31. Januar 1894 (SR 131.218); Art. 35 Abs. 2 der Verfassung des Kantons Solothurn vom 8. Juni 1986 (SR 131.221); § 32 Abs. 3 der Verfassung des Kantons Basel-Landschaft vom 17. Mai 1984 (SR 131.222.2); Art. 35 Abs. 1 der Verfassung des Kantons Schaffhausen vom 17. Juni 2002 (SR 131.223). Daneben können vereinzelte Kantonsparlamente zu Abstimmungsvorlagen eine Variante vorschlagen; siehe ferner Hangartner/Kley (Fn. 36), N. 1711, 1726, 1734, 2355 f.

127 Vgl. Sснӧснцi (Fn. 122), S. 21.

128 Vgl. vorne Fn. 92. 


\section{Zusammenfassende Schlussfolgerungen}

[35] Im Bereich des Dringlichkeitsrechts herrscht ein offensichtliches Spannungsverhältnis: Einerseits besteht die Notwendigkeit, gesetzgeberisch rasch zu handeln, anderseits die Forderung, die direktdemokratischen Rechte möglichst weitgehend zu wahren. Der Auflösung dieses Spannungsverhältnisses dient das 1949 aufgrund einer Volksinitiative eingeführte nachträgliche Referendum im Bund.

[36] Die Bundesversammlung machte, wie eine Untersuchung ihrer Praxis zeigt, auch nach der Einführung des nachträglichen Referendums ziemlich regen Gebrauch vom Dringlichkeitsrecht wenn auch nach Perioden in sehr unterschiedlichem Ausmass. Volksabstimmungen waren bisher jedoch selten, weil nur in wenigen Fällen ein obligatorisches Referendum wegen fehlender Verfassungsgrundlage des Erlasses notwendig war und ansonsten nur ganz vereinzelt das fakultative Referendum ergriffen wurde. Kam es aber doch einmal zu einer Volksabstimmung, so wurden die Vorlagen bis auf eine einzige Ausnahme durchwegs angenommen, was sich mit dem Problemdruck in Fällen dringlicher Rechtsetzung und der Status-quo-Verzerrung gerade in unsicheren Zeiten erklären lässt.

[37] Mit dem Covid-19-Gesetz erreichte die dringliche Gesetzgebung nun aber eine ganz neue "Qualität», indem dieses - anders als die bisherigen dringlichen Erlasse - nicht die Rechtsgrundlage für die punktuelle Bekämpfung eines dringlichen Einzelproblems, sondern das rechtliche Fundament für die generelle Bewältigung eines Grossteils der gegenwärtigen Krise darstellt und - historisch einmalig - schon vor der Volksabstimmung wieder geändert wurde. Diese Konstellation lässt das nachträgliche Referendum als Einrichtung, um dringliche Gesetzgebung und direkte Demokratie miteinander zu «versöhnen», bis zu einem gewissen Grad ins Leere laufen und wirft kaum befriedigend lösbare rechtliche Probleme auf. Grund dafür ist nicht zuletzt die zweifelhafte Einheit der Materie des Covid-19-Gesetzes. Aufgetrennt in mehrere, auch referendumstechnisch voneinander unabhängige Einzelvorlagen könnte dem Bedürfnis nach direktdemokratischer Kontrolle besser, vor allem differenzierter, Rechnung getragen werden.

Stefan G. Schmid, Prof. Dr. iur., ist Ordinarius für Öffentliches Recht mit Schwerpunkt Verfassungsrecht an der Universität St. Gallen.

Micha Herzog, M.A. HSG in Law, Rechtsanwalt, und Dumenig Stiffler, B.A. HSG in Law \& Economics, sind Assistenten am Lehrstuhl von Prof. Schmid. 